\title{
Contribuição ao estudo da anatomia macroscópica dos rins e das estruturas vasculares sanguíneas no hilo renal do bicho-preguiça (Bradypus variegatus)
}

\author{
[Contribution to the study of macroscopic anatomy of the kidneys and blood vascular structures of \\ the renal hilum of sloth (Bradypus variegatus)]
}

\section{"Artigo Científico/Scientific Article"}

\author{
Felipe Coral dos Santos ${ }^{1 *}$, Lucilo Bioni da Fonsêca Filho ${ }^{2}$, Priscilla Virginio de Albuquerque ${ }^{1}$, \\ Júlio Cézar dos Santos Nascimento ${ }^{3}$, Silvia Fernanda de Alcantara ${ }^{4}$, \\ Maria Eduarda Coelho de Miranda ${ }^{4}$, Gilcifran Prestes de Andrade ${ }^{1}$, \\ Marleyne José Afonso Accioly Lins Amorim ${ }^{1}$
}

\begin{abstract}
${ }^{1}$ Departamento de Morfologia e Fisiologia Animal, Universidade Federal Rural de Pernambuco, Recife-PE, Brasil. ${ }^{2}$ Departamento de Medicina Veterinária, Universidade Federal Rural de Pernambuco, Recife-PE, Brasil.

${ }^{3}$ Departamento de Zootecnia, Universidade Federal Rural de Pernambuco, Recife-PE, Brasil.

${ }^{4}$ Departamento de Biologia, Universidade Federal Rural de Pernambuco, Recife-PE, Brasil.

*Autor para correspondência/Corresponding author: E-mail: coral.felipe@ gmail.com
\end{abstract}

\section{Resumo}

Devido à ação antrópica sob o habitat natural do Bradypus variegatus, esta espécie encontra-se em risco de extinção. Um dos principais pontos para a conservação de espécies em extinção é conhecer sua anatomia e fisiologia. Porém, para o B. variegatus, com hábito exclusivamente herbívoro, que se alimenta de folhas, flores, brotos, talos verdes e frutos, estas sendo sua principal fonte de ingestão hídrica, falta literatura sobre o funcionamento e aspectos anatômicos de seus rins. Logo, realizou-se um estudo da anatomia macroscópica dos rins e de suas estruturas vasculares, que atravessam o hilo renal. O objetivo é avaliar se sua estrutura possui diferenças anatômicas que justifiquem o baixo consumo de água. Oito pares de rins provenientes de bichospreguiça adultos, pertencentes ao acervo do Departamento de Morfologia e Fisiologia Animal (DMFA) da Universidade Federal Rural Pernambuco, foram utilizados para o estudo. Os rins foram retirados, dissecados, identificados e avaliados macroscopicamente. Os rins em B. variegatus apresentam forma elíptica, semelhante a um grão de feijão. Possui uma margem côncava e lisa, enquanto a outra é convexa, na qual se faz presente o hilo renal, com a presença das estruturas vasculares. Não foram observadas divisões em lobos, sendo classificado como órgão unilobado liso.

Palavras-chave: anatomia; extinção; herbívoro; hilo renal; mamíferos.

\begin{abstract}
Because of anthropic influence on the natural habitat of Bradypus variegatus, this species is at a high risk of extinction. One of the key points in conservation of an endangered species is knowing its anatomy and physiology. However, for B. variegatus, who is entirely herbivorous and feeds on leaves, flowers, buds, green stalks, and fruits, with these being its only water source, there is a lack of information in the literature pertaining to the function and anatomy of its kidneys. Therefore, a study on the macroscopic anatomy of the kidney and its vascular structures, which cross the renal hilum, was performed. The objective was to evaluate if the sloth kidney had anatomical differences that would justify the low water consumption. Eight pairs of kidneys from adult sloths belonging to the collection of the Department of Animal Morphology and Physiology (DMFA) of UFRPE were used in this study. The kidneys were removed, dissected, identified, and evaluated macroscopically. B. variegatus kidneys have an elliptical shape, similar to a bean. It has a concave smooth margin and a convex margin where the renal hilum and vascular structures are located. The kidney was not divided into lobes, and was thus classified as a smooth, unilobar organ.
\end{abstract}

Keywords: anatomy; extinction; herbivore; renal hilus; mammals. 


\section{Introdução}

Os bichos-preguiça pertencem à Ordem Pilosa estando distribuídos em duas famílias mediante a quantidade de dedos nos membros torácicos, sendo a família Bradypodidae, gênero Bradypus, bichos-preguiça com três dedos nestes membros e a família Megalonychidae, gênero Choloepus, com dois dedos. Particularmente, o bicho-preguiça de três dedos e de pescoço marrom, muito encontrado no Brasil, foi descrito como Bradypus variegatus (Gardner, 2005).

Com o avanço das fronteiras agrícolas, o meio ambiente está sendo fortemente alterado proporcionando significativas mudanças à fauna $\mathrm{e}$ à flora, ameaçando o habitat natural de muitas espécies (Bianchi et al., 2012).

Os bichos-preguiça são mamíferos neotropicais, com hábito arborícola e prioritariamente herbívoro (Wilson e Deeann, 2005). Dentre esses mamíferos, podemos destacar B. variegatus com maior distribuição, estando presente de Honduras até o sudeste brasileiro, todavia apresenta uma difícil adaptação em cativeiro (Montgomery e Sunquist, 1975; MoraisBarros et al., 2006).

O Brasil é o país habitat detentor da maioria das espécies de bichos-preguiça vivos (Wetzel e Kock, 1973; Cherem et al., 2004). Dessa forma, o país ocupa uma posição de maior reserva natural de bradipodídeos, o que permite que esses animais sejam vistos como modelos biológicos para as pesquisas de caráter multidisciplinar, permitindo deste modo a continuidade de estudos sobre filogenia, anatomia, histologia, patologia, ecologia desses mamíferos tão peculiares (Amorim et al., 2003). Dos representantes da Ordem Pilosa, os bichos-preguiça apresentam o menor índice de ingestão de água, sendo o consumo hídrico restrito à água das folhas, frutos e legumes que se alimentam, assim, é importante verificar se esses animais possuem alguma diferença morfológica ou fisiológica, que possa justificar ou compensar o baixo consumo de água.

Diante desse contexto, a presente pesquisa teve por objetivo contribuir com o estudo da anatomia e distribuição topográfica dos elementos vasculares sanguíneos na região extra hilar dos rins de $B$. variegatus, a fim de tornar mais clara as características morfofisiológicas dessa espécie. $\mathrm{O}$ rim, principal órgão excretor, já foi estudado no gênero Bradypus, especificamente em Bradypus torquatus, porém, ainda não há estudos que relatem esse órgão no bicho-preguiça de pescoço marrom e há muito a ser elucidado a respeito da anatomia, histologia e fisiologia da espécie em questão. Neste sentido, os resultados poderão ser utilizados para fins de comparação de aspectos morfológicos e evolutivos entre $B$. variegatus e outros mamíferos, além de contribuírem para a valorização desse animal no cenário atual onde ainda são desconhecidos por muitos, e principalmente aumentar as preocupações com a conservação e preservação desses eutérios.

\section{Material e Métodos}

Para este trabalho, foram utilizados oito bichos-preguiça adultos da espécie $B$. variegatus, todos obtidos após morte natural, de onde foram retirados os oito pares de rins.

Todos os animais utilizados fazem parte do acervo do Laboratório de Anatomia do Departamento de Morfologia e Fisiologia Animal da Universidade Federal Rural de Pernambuco (DMFA-UFRPE) e foram fixados em solução de formaldeído a $20 \%$ estando mantidos em tanque de igual solução. Os espécimes apresentaram cavidade abdominal completa, permitindo a localização exata dos rins.

Os cadáveres foram dissecados, a partir de uma incisão sagital mediana e transversal do abdômen para a descrição da esqueletopia e sintopia dos rins. Para uma melhor descrição da topografia dos rins, foram delimitados quadrantes abdominais com linha zero pré-lavada, sendo estas: crânioventral, crâniodorsal, caudoventral e caudodorsal.

Uma linha imaginária foi disposta em sentido longitudinal entre os polos cranial e caudal do rim, enquanto a outra foi disposta no sentido dorsoventral, sendo perpendicular à primeira, cruzando-se no centro do hilo renal, ao nível do ureter. A partir desse traçado, determinaram-se as regiões hilar, justahilar e extrahilar. Essas delimitações permitiram uma melhor observação da posição anatômica dos vasos presentes no hilo renal.

Após a análise preliminar da localização, foi feita a individualização dos rins, sendo estes devidamente identificados e submetidos à minuciosa avaliação, obtendo-se suas medidas anatômicas externas como largura, espessura e comprimento, a partir do emprego de um paquímetro manual $(150 \mathrm{~mm}-0,02 \mathrm{~mm})$. Foram dissecadas também a artéria renal e a veia renal de cada rim. Todos os resultados foram fotografados. 
E foi utilizada a terminologia anatômica de acordo com International Commitee on Veterinary Gross Anatomical Nomeclature (2012).

\section{Resultados}

Os dados da análise macroscópica desse estudo revelaram as dimensões anatômicas dos rins de $B$. variegatus, as quais foram obtidas por meio de mensurações com paquímetro manual (Tabela $1)$.

Os rins dessa espécie apresentaram-se com aspecto de grão de feijão. Em relação à anatomia externa, os animais apresentaram em média $54 \mathrm{~cm}$ de comprimento crâniocaudal.

Tabela 1. Mensuração dos rins do bicho-preguiça (Bradypus variegatus) em centímetros.

\begin{tabular}{lcccccccc}
\hline \multicolumn{1}{c}{ Animal } & $\begin{array}{c}1 \\
\text { Macho }\end{array}$ & $\begin{array}{c}2 \\
\text { Fêmea }\end{array}$ & $\begin{array}{c}3 \\
\text { Macho }\end{array}$ & $\begin{array}{c}4 \\
\text { Fêmea }\end{array}$ & $\begin{array}{c}5 \\
\text { Macho }\end{array}$ & $\begin{array}{c}6 \\
\text { Macho }\end{array}$ & $\begin{array}{c}7 \\
\text { Fêmea }\end{array}$ & $\begin{array}{c}8 \\
\text { Fêmea }\end{array}$ \\
\hline Rim Direito & & & & & & & & \\
Comprimento & 2,5 & 3,5 & 2,6 & 2,4 & 2,5 & 2,3 & 3,3 & 2,2 \\
Largura & 1,9 & 2,5 & 1,5 & 1,2 & 1,5 & 1,7 & 2,0 & 1,4 \\
Espessura & 1,6 & 2,0 & 1,2 & 1,0 & 1,1 & 1,3 & 1,8 & 1,1 \\
Rim Esquerdo & & & & & & & & \\
Comprimento & 2,9 & 3,4 & 2,3 & 2,1 & 2,2 & 2,1 & 2,8 & 2,0 \\
Largura & 1,6 & 2,3 & 1,7 & 1,5 & 1,7 & 1,5 & 1,9 & 1,5 \\
Espessura & 1,3 & 1,6 & 1,3 & 1,2 & 1,2 & 1,3 & 1,5 & 1,3 \\
\hline
\end{tabular}

Quanto aos rins, estes órgãos apresentaram uma superfície lisa, mostrando aspecto unilobado (não há divisões por lobos visíveis), não sendo feita a análise do aspecto da coloração, uma vez que os animais foram formolizados. Lateralmente, o rim possui uma margem convexa e em sua margem medial uma superfície côncava, onde se localiza o hilo renal, entrada e saída para vasos sanguíneos, nervos e pelve renal. $\mathrm{O}$ hilo renal possui formato elipsoide e apresentou as estruturas comuns às outras classes de mamíferos, como veia renal, artéria renal e ureter. Ao corte coronal, que divide o rim em duas partes iguais, a análise interna confirmou que o rim dessa espécie não se apresenta dividido em lobos, permitindo observar que existem duas regiões bem distintas, chamada de córtex renal, com distribuição mais periférica e cor pálida e a medula, disposta de forma mais central e com coloração mais escura (Figura 1).

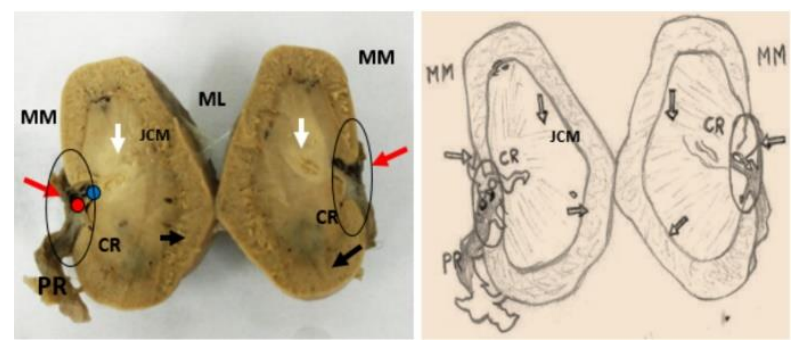

Figura 1. Fotomacrografia e desenho esquemático do parênquima renal de bicho-preguiça (Bradypus variegatus) em corte coronal. Córtex (setas pretas) e medula (setas brancas), hilo renal (setas vermelhas), junção cortéx-medular (JCM), pelve renal (PR), margem lateral (ML), margem medial $(\mathrm{MM})$, crista renal $(\mathrm{CR})$; artéria renal (círculo vermelho) e veia (círculo azul).
Em relação ao posicionamento anatômico, os rins de $B$. variegatus estão situados acima da cintura pélvica entre o peritônio e a parede posterior do abdômen, assentando-se em duas impressões que os acomodam em posição retroperitoneal (Figura 2A). A contagem das vértebras permitiu descrever com precisão que os rins dessa espécie se localizam na altura entre a L3 e L5, sendo o rim direito mais cranial que o esquerdo.

Essa configuração resulta numa impressão renal no lobo direito do fígado. A análise confirmou que os rins nessa espécie são órgãos pares e com formato de grão de feijão (Figura 2B). As avaliações dimensionais revelaram que o comprimento médio do rim direito é de $2,7 \mathrm{~cm}$, enquanto a largura média é de $1,8 \mathrm{~cm}$ e, a espessura média é de 1,4 cm. Todavia, para o rim esquerdo, os resultados demonstraram em média $2,5 \mathrm{~cm}$ de comprimento, $1,7 \mathrm{~cm}$ de largura e $1,3 \mathrm{~cm}$ de espessura (Figura 2C).

No rim direito, assim como no esquerdo, constatou-se a presença de dois ramos venosos, um cranial e um caudal ao hilo renal, os quais são ramos tributários da veia renal. As veias e artérias foram observadas em quadrantes diferentes. Os ramos arteriais e venosos no rim esquerdo foram observados em $75 \%$ dos casos no quadrante crânioventral, enquanto $25 \%$ se localizaram no quadrante crâniodorsal. No rim direito, $75 \%$ das veias e artérias foram observados no quadrante crâniodorsal e $25 \%$ no quadrante crânioventral. Não obtivemos proporções iguais entre os quadrantes ventrais e dorsais em nenhum animal. Os vasos arteriais e venosos demonstraram uma 
localização predominantemente craniana, $100 \%$ dos animais apresentaram esse perfil, não havendo artérias e veias nos quadrantes caudais em nenhum dos indivíduos. A pelve renal foi observada em todos os animais $(100 \%)$ predominantemente nos quadrantes caudais, sendo, em $75 \%$ dos espécimes, o ureter encontrado entre os quadrantes caudoventral e caudodorsal.

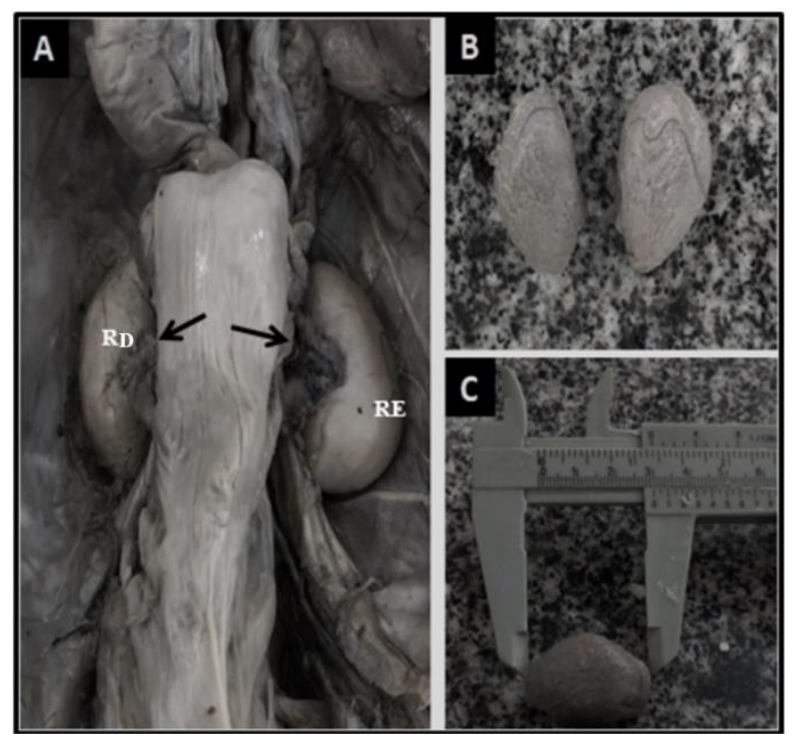

Figura 2. Fotomacrografia dos rins de bicho-preguiça (Bradypus variegatus). A) Vista ventral dos rins. B) Formato característico de grão de feijão dos rins. C) Medidas com auxílio de paquímetro manual. Rim direito (RD), rim esquerdo (RE) e o hilo renal (setas).

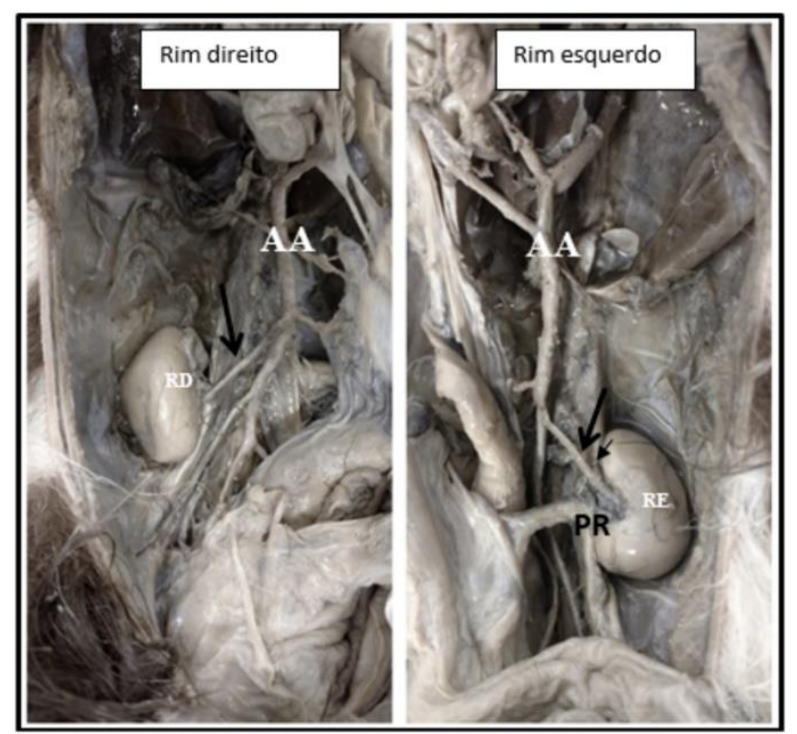

Figura 3. Fotomacrografia da cavidade abdominal do bichopreguiça (Bradypus variegatus). Artéria aorta (AA), artérias renais (setas longas), veia renal (seta curta), pelve renal (PR).

Em grande parte dos animais analisados, as veias e artérias prevaleceram na região hilar, tanto no rim direito, quanto no rim esquerdo, observando-se então, uma localização predominantemente central tanto para artérias quanto para veias renais. Nas regiões justahilar e extrahilar foram observados os prolongamentos dos vasos sanguíneos, porém, todos partindo da região hilar. As artérias e as veias renais apresentaram-se igualmente distribuídas na maioria dos espécimes, em ambos os rins, direito e esquerdo. Observou-se em dois animais, uma localização justahilar das artérias e veias renais em ambos os rins (Figura 4).

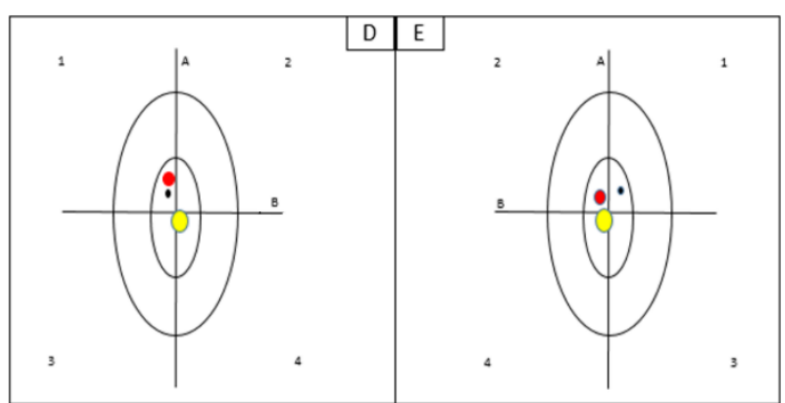

Figura 4. Representação esquemática do padrão de localização dos segmentos renais de bichopreguiça (Bradypus variegatus). (D) Rim direito, (E) Rim esquerdo; (A) Linha craniocaudal, (B) Linha dorsoventral; (1) Quadrante crâniodorsal, (2) Quadrante crânioventral, (3) Quadrante caudodorsal, (4) Quadrante caudoventral; ureter (círculo amarelo), artéria renal (círculo vermelho), veia renal (círculo preto).

As diferenças encontradas em relação à anatomia e à posição dos rins não são significativamente expressivas, sendo avaliadas como variações próprias de alguns dos animais. Em relação à localização das veias e artérias, diferenças regionais não foram acentuadamente notadas, estando grande parte dos espécimes analisados em conformidade com o padrão encontrado.

\section{Discussão}

Os rins de $B$. variegatus são órgãos retroperitoneais e estão envoltos por uma cápsula de tecido conjuntivo e divididos em duas camadas, a cortical e a medular semelhante ao que foi descrito para o bicho-preguiça-de-coleira ( $B$. torquatus) (Bianchi et al., 2012). Os rins de $B$. variegatus possuem formato semelhante a um grão de feijão o que se assemelha ao que foi descrito também para B. torquatus (Bianchi et al., 2012) e para Gracilinanus microtarsus (Prado et al., 2014). Os rins apresentaram uma superfície lisa e sem divisão em lobos, sendo classificados como unilobar liso, além disso, demonstrou lateralmente 
uma margem convexa sem estruturas presentes e medialmente uma margem côncava, onde foram visualizados o hilo renal, porta de entrada e saída para vasos sanguíneos e a pelve renal, respectivamente, aspectos esses que corroboram com o que foi descrito para cuícas da espécie $G$. microtarsus (Prado et al., 2014), mas diferem dos achados em búfalos, que possuem rins multilobados (Menezes et al., 2014).

O rim direito foi observado mais cranialmente em relação ao rim esquerdo, sendo ambos localizados atrás da parede abdominal de forma retroperitoneal, esse resultado é semelhante ao encontrado para cuícas (Prado et al., 2014) e alguns carnívoros domésticos (Konig e Liebich, 2011).

Em B. variegatus, espécie que tem predominância em regiões tropicais, ou seja, regiões com alta umidade, não foram observadas adaptações anatômicas que possam ser relacionadas ao baixo consumo hídrico, seguindo os padrões encontrados por outros mamíferos. Deve-se ressaltar que esses animais não costumam ingerir água em seu hábitat natural (Malta e Luppi, 2006), mas para animais em cativeiro, foi observado que quando não suprem suas necessidades hídricas, são obrigados a ingerir água das folhas e brotos através de lambeduras (Santos, 1945; Gilmore, 2000).

As artérias renais em $B$. variegatus apresentam-se de forma única em cada antímero, uma direita e uma esquerda, sendo os dois ramos provenientes da aorta, o que é semelhante ao encontrado em coelhos (Barone, 1997) e em capivaras (Romagnolli et al., 2003) e difere do que foi descrito para caprinos (Abidu-Figueiredo, 2009).

Quanto aos ramos cranial e caudal das veias renais, tanto direita quanto esquerda, nota-se que seguem o mesmo percurso e desembocam no respectivo ramo da veia cava caudal. Superior aos rins, estes ramos encontram-se e formam apenas uma veia cava caudal, a qual segue em direção ao átrio direito do coração, informação que confirma os achados para a própria espécie (Barretto et al., 2013).

A origem da artéria renal direita em $B$. variegatus já foi observada através de um único tronco que se trifurca dando origem à artéria renal direita, genital e mesentérica cranial, fato este que corrobora com a ocorrência de artérias renais múltiplas no rim de cães vira-latas (Lanz e Waldron, 2000). Comportamento vascular não visualizado na artéria renal esquerda, que possui também origem direta da artéria aorta. Esse aspecto é de extrema importância para que se evite erros por desconhecimento anatômico em eventuais intervenções cirúrgicas (Oliveira e Guimarães, 2007).

As artérias renais podem diferir de acordo com a distribuição e o arranjo, podendo se apresentar únicas ou múltiplas, os achados da pesquisa demonstraram a presença de apenas uma artéria renal ingressando em cada órgão, assemelhando-se ao que foi descrito para catetos (Machado et al., 2000).

\section{Conclusão}

Os rins de bichos-preguiça (B. variegatus) são lisos, possuem forma de grão de feijão e aspecto unilobado. As artérias e veias renais ocorrem em dose simples e localizam-se na região hilar, enquanto os ramos venosos ocorrem em dose dupla. Conclui-se que a espécie B. variegatus possui anatomia e topografia dos segmentos arteriais e venosos dos rins semelhantes à maioria dos mamíferos.

\section{Conflito de Interesse}

Os autores declaram não existir conflito de interesse.

\section{Comissão de Ética}

O protocolo experimental para a presente pesquisa possui autorização do Comitê de Ética no uso de animais - CEUA da Universidade Federal Rural de Pernambuco. A pesquisa intitulada Estudo anatômico dos rins e das estruturas vasculares nas regiões hilares dos rins no bicho-preguiça $(B$. variegatus) Schinz, 1825, possui licença de número 077/2015, e, faz parte do projeto de maior abrangência intitulado Estudo morfológico dos sistemas orgânicos do bicho-preguiça $B$. variegatus (Schinz, 1825), o qual possui número de licença 034/2015.

\section{Referências}

Abidu-Figueiredo, M.; Roza, M.S.; Passos, N.C.; Silva, B.X.; Scherer, P.O. Artéria renal com dupla origem na porção abdominal da aorta em caprino. Acta Veterinaria Brasilica, 3(1): 3842, 2009.

Amorim, M.J.A.A.L.; Miglino, M.A.; Amorim Júnior, A.A.; Santos, T.C. Aspectos morfológicos da placenta da preguiça, Bradypus variegatus (Shinz, 1825). Brazilian 
Journal of Veterinary Research and Animal Science, 40(3): 217-226, 2003.

Barone, R. Anatomie compare dés mammiféres domestiques. Vol. 3. $2^{\mathrm{a}}$ ed. Paris. 1997. 130 p.

Barretto, M.L.M.; Amorim, M.J.A.A.L.; Falcão, M.V.D. Análise morfológica e morfométrica das gônadas de preguiça (Bradypus variegatus Schinz, 1825). Pesquisa Veterinária Brasileira, 33(9): 1130-1136, 2013.

Bianchi, P.K.F.C.; Cardoso, F.; Salvadori, M.L.B.; Lima, A.R.; Santos, G.M.F.; Kfoury Junior, J.R. Morfologia renal do Bradypus torquatus. Biotemas, 25(4): 201-205, 2012.

Cherem, J.J.; Simões-Lopes, P.C.; Althoff, S.L.; Graipel, M.E. Lista dos mamíferos do Estado de Santa Catarina, sul do Brasil. Mastozoología Neotropical, 11(2): 151-184, 2004.

Gardner, A.L. Order Pilosa. In: Wilson, D.E.; Reeder, D.M. Mammal species of the world. 1. v. Baltimore: Johns Hopkins University Press, 2005. p.100-103.

Gilmore, D.P.; Da Costa, C.P.; Duarte, D.P.F. An update on the physiology of two - and three toed sloths. Brazilian Journal of Medical and Biological Research, 33(2): 129-146, 2000.

König, H.E.; Liebich, H.G. Órgãos urinários (Organa Urinaria). In: König, H.E.; Liebich, H.G. Anatomia dos animais domésticos: texto e atlas colorido. $4^{\mathrm{a}}$ ed. Porto Alegre: Artmed. 2011. p.411-425.

Lanz, O.I.; Waldron, D.R. Renal and ureteral surgery in dogs. Clinical Techniques in Small Pratice, 15(1): 1-10, 2000.

Machado, G.V.; Cavalcante Filho, M.F.; Miglino, M.A.; Carvalho, M.A.M.; Santos, T.C.; Lesnau, G.G. Comportamento anatômico das artérias renais em catetos (Tayassu tajacu Linnaeus, 1758). Veterinária Notícias, 6(1): 17-25, 2000.

Malta, M.C.C.; Luppi, M.M. Marsupialia Didelphimorphia (gambá, cuíca). In: Cubas, Z.S.; Silva, J.C.R.; Catão-Dias, J.L. Tratado de animais selvagens: medicina veterinária. São Paulo: Roca, 2006. p.340-357.
Menezes, D.J.A.; Ferraz, R.H.S.; Santos, J.R.S.; Silva, F.E.; Neto, R.B.S.; Vianna, E.L. Aspectos anatômicos do rim de búfalos. Archives of Veterinary Science, 19(4): 72-77, 2014.

Montgomery, G.G.; Sunquist, M.E. Impact of sloths on neotropical energy flow and nutrient cycling. In: Golley, F.; Medina, E. Tropical ecological systems: trends in terrestrial and aquatic research. New York: Springer-Verlag, 1975. p.69-98.

Moraes-Barros, N.; Silva, J.A.; Miyaki, C.Y.; Morgante, J.S. Comparative phylogeography of the atlantic forest endemic sloth (Bradypus torquatus) and the widespread three-toed sloth (Bradypus variegatus) (Bradypodidae, Xenarthra). Genética, 126: 189-98, 2006.

Oliveira, F.S.; Guimarães, G.C. Duplicidade de artéria renal em cão. Ciência Rural, 37(6): 1817-1819, 2007.

Prado, C.V.G.B.; Carniatto, C.H.O.; Santos, A.C.M.; Rodrigues, R.F.; Favaron, P.O.; Rodrigues, M.N.; Miglino, M.A. Arquitetura e estrutura dos rins de Gracilinanus microtarsus (Didelphimorphia: Didelphidae) (Wagner, 1842). Brazilian Journal of Veterinary Research and Animal Science, 51(4): 297303, 2014.

Romagnolli, P.; Machado G.V.; Miglino M.A. Segmentos arteriais dos rins de queixadas (Tayassu pecari Link, 1795). Brazilian Journal of Veterinary Research Animal Science, 40(3): 205-212, 2003.

Santos, E. Entre o gambá e o macaco: vida e costumes dos mamíferos no Brasil. 1. ed. Rio de Janeiro: Editora F. Bryiet \& Cia. 1945. 287p.

Wetzel, R.M.; Kock, D. The identity of the Bradypus variegatus Schinz (Mammalia: Edentata). Proceeding of Biological Society, 86: 25-34, 1973.

Wilson, D.; Deeann M.R. Bradypus variegatus. Mammal species of the world: a taxonomic and geographic reference. $3^{\text {rd }}$ ed. Baltimore: Johns Hopkins University Press. 2005. 2142p. 